\title{
The Clinical Relevance of Stromal Matrix Metalloproteinase Expression in Ovarian Cancer
}

\author{
Aparna A. Kamat ${ }^{1}$, Mavis Fletcher ${ }^{4}$, Lynn M. Gruman ${ }^{4}$, Peter Mueller ${ }^{2}$, Adriana Lopez ${ }^{2}$, \\ Charles N. Landen Jr. ${ }^{1}$, Liz Han ${ }^{1}$, David M. Gershenson ${ }^{1}$, and Anil K. Sood ${ }^{1,3}$ \\ ${ }^{1}$ Department of Gynecologic Oncology, University of Texas M.D. Anderson Cancer Center, \\ Houston, Texas \\ ${ }^{2}$ Department of Biostatistics and Applied Mathematics, University of Texas M.D. Anderson \\ Cancer Center, Houston, Texas \\ ${ }^{3}$ Department of Cancer Biology, University of Texas M.D. Anderson Cancer Center, Houston, \\ Texas \\ ${ }^{4}$ Department of Pathology, University of lowa Hospitals and Clinics, lowa City, lowa
}

\begin{abstract}
Purpose-Matrix metalloproteinases (MMP) are proteolytic enzymes implicated in cancer progression and metastasis. We sought to determine the role of epithelial (tumor cell - derived) and stromal (host-derived) expression of MMPs in predicting the clinical outcome of patients with epithelial ovarian cancer (EOC).
\end{abstract}

Experimental Design-MMP-2, MMP-9, and membrane type 1 (MT1)-MMP expression was evaluated using immunohistochemistry in 90 invasive EOCs, and samples were scored for epithelial and stromal staining. Results were correlated with clinicopathologic characteristics using univariate and multivariate analyses.

\begin{abstract}
Results-High expression of MMP-2, MMP-9, and MT1-MMP in tumor epithelium was detected in 54\%, 97\%, and 100\% of cases, and in stromal compartments, in 38\%, 70\%, and $38 \%$ of cases, respectively. High stromal expression of MMP-2, MMP-9, and MT1-MMP was significantly associated with aggressive features such as high stage, high grade ascites, and positive lymph node status. Kaplan-Meier analysis showed that high epithelial and stromal expression of MMP-2, MMP-9, and MT1-MMP were each significantly associated with shorter disease-specific survival (DSS; $P<0.01$ ). On tree-structured survival analysis, patients with strong epithelial MT1-MMP expression had the shortest DSS, whereas patients with moderate epithelial MT1-MMP and low stromal MMP-9 expression had the longest DSS $(P<0.01)$. On multivariate analysis, high stromal expression of MMP-9 $(P=0.01)$ andMT1-MMP $(P=0.04)$, strong epithelial MT1-MMP $(P=0.01)$ and high stage $(P=0.04)$ were independent predictors of poor DSS.
\end{abstract}

Conclusions-Overexpression of stromal MMP-9 and MT1-MMP is independently associated with shorter DSS in EOC. Thus, host-derived MMPs are valuable predictors of clinical outcome in EOC.

\footnotetext{
(C) 2006 American Association for Cancer Research

Requests for reprints: Anil K. Sood, Departments of Gynecologic Oncology and Cancer Biology, The University of Texas M.D. Anderson Cancer Center, Unit 1362, P.O. Box 301439, Houston, TX 77230-1439. Phone: 713-745-5266; Fax: 713-792-7586; asood@mdanderson.org..

Note: Presented at the 36th Annual Meeting of the Society of Gynecologic Oncologists, Miami, FL, March 20-23, 2005.
} 
Ovarian cancer remains the leading cause of death from a gynecologic malignancy in the U.S. (1). Epithelial ovarian carcinomas (EOC) account for nearly $90 \%$ of all ovarian malignancies, and most patients present with widely metastatic disease at diagnosis, contributing to the high mortality associated with this disease (2). Local invasion and distant spread of malignant neoplasms involves degradation of subepithelial and subendothelial basement membranes and modification of the extracellular matrix (ECM; ref. 3). It is now known that this process depends on a series of complex interactions between tumor cells, host-derived stromal cells, and endothelial cells. These interactions are mediated by a number of factors including proteolytic enzymes, adhesion molecules, transcriptional factors, and components of the ECM (4).

Matrix metalloproteinases (MMP) comprise a large family of zinc- and calcium-dependent proteolytic enzymes that have been repeatedly implicated in invasion and metastasis. MMPs are capable of degrading most components of the ECM, including the basement membrane, which serves as a barrier between tissue compartments (5). Disruption of basement membrane integrity, a feature of invasive tumors, allows tumors to spread locally and to distant sites (6). MMP-2 and MMP-9 degrade type IV collagen, a major component of the basement membrane, and are associated with active neovascularization (7). Higher mRNA, protein and activity levels of both MMP-2 and MMP-9 have been shown in ovarian carcinomas compared with normal ovaries, benign, or borderline tumors (8-10). Increased expression of these type IV collagenases has been positively correlated with disease progression (11), and has been negatively correlated with survival (12) in ovarian cancer. A subset of MMPs, known as membrane-type MMPs (MT-MMP) contain a transmembrane domain, and unlike other MMPs, are not secreted, but remain attached to cell surfaces (13). MT1-MMP cleaves pro-MMP-2 and is the key activator of MMP-2 (14). In ovarian carcinomas and in malignant effusions, a close association between MMP-2 and MT1-MMP has been well described $(15,16)$. Based on the known functional significance of MMP-2, MMP-9, and MT1-MMP in ovarian carcinomas, we focused on these MMPs in the present study.

MMPs can be produced by tumor cells as well as the surrounding stromal cells, such as fibroblasts, infiltrating macrophages, and endothelial cells (17). Host-derived MMP-9 expression in tumor-infiltrating macrophages has been shown to play a critical role in angiogenesis and progressive growth of human ovarian tumors in mice (18). Similarly, stromal cells express higher levels of MMP-2 as compared with tumor cells in a variety of malignant tumors including breast and gastric carcinomas $(19,20)$. A recent study showed that stromal MMP-2 expression was an important predictor of recurrence-free survival in patients with endometrioid ovarian adenocarcinomas (21). However, whereas most studies have investigated the role of tumor cell-derived expression of MMPs, there is limited data regarding the clinical relevance of stromal MMPs in EOC. Therefore, the purpose of our study was to determine the prognostic significance of epithelial (tumor cell-derived) and stromal (host-derived) expression of MMP-2, MMP-9, and MT1-MMP in patients with EOC.

\section{Materials and Methods}

\section{Samples for immunostaining}

Following Institutional Review Board approval, archived formalin-fixed, paraffin-embedded samples were obtained from 90 patients with primary EOC who were surgically treated at the Holden Comprehensive Cancer Center, University of Iowa Hospitals and Clinics, Iowa City, IA between 1990 and 2000. All patients were surgically staged based on the International Federation of Gynecology and Obstetrics (FIGO) staging system. The study included 60 patients with serous and 30 patients with nonserous adenocarcinomas. 


\section{Immunohistochemical staining}

Formalin-fixed paraffin-embedded samples were sectioned at $4 \mu \mathrm{m}$ and stained with $\mathrm{H} \& \mathrm{E}$ for tumor confirmation. Sections adjacent to the H\&E staining were used for immunohistochemical staining. All slides were deparaffinized with xylene, $100 \%$ ethanol and $95 \%$ ethanol, followed by a thorough deionized water wash. Antigen recovery was done using a digestion antigen recovery technique with Ficin (Zymed, San Francisco, CA) at $37^{\circ} \mathrm{C}$ for 5 minutes on all slides. For MMP-9 and MT1-MMP, water bath antigen recovery technique [Retrievit (pH 8), Innogenex, San Ramon, CA] was done on all slides. Immunohistochemical staining was done using the Dako Autostainer (Dako Corp., Carpinteria, CA) using the peroxidase kit (Vectastain Universal Elite ABC, PK-7200; Vector Laboratories, Inc., Burlingame, CA) to detect mouse anti-human MMP-2 (dilution 1:50; Labvision Neomarkers, Fremont, CA), mouse anti-human MMP-9 (dilution 1:40, Oncogene Research Products, Boston, MA), or rabbit anti-human MT1-MMP (dilution 1:275, Labvision Neomarkers). The antibody was diluted with an antibody diluent (Dako). Following deparaffinization and antigen recovery, slides were washed in Tris-buffered $\mathrm{NaCl}$ solution with Tween (TBST). Slides were blocked in three steps: $0.03 \%$ hydrogen peroxide (Dako) for 20 minutes followed by a TBST wash, avidin and biotic blocks for 10 minutes each followed by a TBST wash after each step, and finally the serum-free protein block (Dako) for 15 minutes. Slides were incubated with primary antibody for 45 minutes (MMP-2), 100 minutes (MMP-9), and 90 minutes (MT1-MMP). Slides were rinsed in TBST, followed by application of the Vectastain secondary antibody for 20 to 30 minutes. This was followed by a TBST wash and incubation with the Ready-to-Use avidin-biotin complex method reagent. Color for the Ready-to-Use reagent was produced using substrate (3,3'-diaminobenzidine + Brown, K3468, Dako) for 5 to 15 minutes. Slides were counterstained with Mayer's hematoxylin for 35 to 60 seconds.

\section{Semiquantitative analysis of immunostaining}

All samples were reviewed by two independent investigators (M.S. Fletcher and A.K. Sood), who were blinded to the clinical outcome of patients. Serial sections of the tumor blocks from the primary tumor were evaluated for immunohistochemical analysis and MMP expression was studied in both tumor cells and stromal cells. Semiquantitative assessment of MMP expression was done as previously described (22) by assessing the percentage of stained tumor and stromal cells and staining intensity. The independent scores from both investigators were consolidated into a final score, which is reported in this study. Any differences in the scores were resolved following discussion between the two investigators.

Briefly, the percentage of positively stained cells was rated as follows: 0 points, $0 \%$ to 5\%; 2 points, $6 \%$ to $50 \% ; 3$ points, $>50 \%$. The staining intensity was rated in the following manner: 1 point, weak intensity; 2 points, moderate intensity; 3 points, strong intensity. Points for the intensity and percentage of positive cells were added, and an overall score (OS $0-3)$ was assigned. Tumors were categorized into four groups based on the OS: negative expression (OS, 0 or $<5 \%$ cells stained regardless of intensity); weak expression (OS, 1) 1 to 2 points; moderate expression (OS, 2) 3 to 4 points; strong expression (OS, 3 ) 5 to 6 points. For statistical analysis, the patients were dichotomized into two groups: low expression (OS, 0 or 1) included those with negative or weak expression and high expression (OS, 2 or 3 ) included those with moderate or strong expression. For epithelial MMP-9 and MT1-MMP, $97 \%$ and $100 \%$ of the samples, respectively, showed high protein expression. In these two groups, samples with moderate or low expression were compared to those with strong expression. 


\section{Clinicopathologic variable analysis}

All patients underwent surgical exploration and primary surgical cytoreduction as the initial treatment. The treating gynecologic oncologist determined the adjuvant therapy. Standard chemotherapy including a platinum-based regimen was given to all patients who received adjuvant chemotherapy. The pathologic diagnosis was verified at the institutional gynecologic oncology tumor board. A gynecologic pathologist (M.S. Fletcher) reviewed all the H\&E slides to confirm the histopathologic diagnosis and tumor grading.

Based on FIGO stage, patients were divided into two groups, low stage (FIGO stage I and II; $n=18$ ) and high stage (FIGO stage III and IV, $n=72$ ). A clinical remission was defined as no evidence of disease based on physical examination, imaging studies and CA 125 levels. Optimal cytoreduction was defined as $<1 \mathrm{~cm}$ of residual disease at the end of surgery. Disease-specific survival (DSS) was defined as the time from entry into the study until the date of death or the date of last contact.

\section{Statistical analysis}

$\chi^{2}$ or Fisher's exact tests were used, as appropriate, to test for the association in the proportions across levels of a single covariate factor and MMP expression. Patients who were alive at last follow-up or died from causes other than ovarian cancer were censored at the date of the last follow-up. The survival function of DSS was estimated using the KaplanMeier product limit method. A two-sided log-rank test was used to test for differences between survival curves. A tree structured survival analysis (23) was done to determine whether any specific combination of MMP expression would confer the poorest outcome. Formal tests were done to validate the regression tree to fit the survival data as a function of the immunohistochemical markers. A log-rank test was used to compare the survival distributions of the subpopulations in the survival tree. DSS of patients presenting with epithelial and stromal expressions of MMP-2, MMP-9, and MT1-MMP and other clinical variables were assessed using both univariate and multivariate Cox proportional hazards regression (24). Hazard ratio (HR) estimates were reported based on this model. $P<0.05$ was considered statistically significant. Statistical analyses were carried out using SAS software (version 8.02, SAS Institute, Cary, NC) and S-Plus software (version 6.1, Insightful Corp., Seattle, WA).

\section{Results}

\section{Patient clinical data}

To determine the association between specific MMPs in human ovarian cancers and diseasespecific clinical outcome, 90 epithelial ovarian carcinomas were stained for MMP-2, MMP-9, and MT1-MMP. The demographic features of the patients in this study are listed in Table 1. Eighty percent of patients had advanced stage disease, and 59\% of tumors were high grade. Serous adenocarcinomas comprised $70 \%$ of all tumors, and $70 \%$ of all patients underwent optimal cytoreduction at the time of primary surgery.

\section{Association of MMP protein expression with clinical and pathologic features}

We first characterized the expression patterns of all three MMPs in the epithelial and stromal compartments. Representative staining patterns for each MMP are illustrated in Fig. 1. Low epithelial MMP-2 expression was present in $45 \%$ of samples, whereas $55 \%$ had high positivity. Stromal MMP-2 expression was detected in 65 of 90 specimens (low expression in $62 \%$ and high expression in 38\%). The presence of strong epithelial MMP-2 expression was significantly associated with positive lymph node status $(P<0.01)$. However, high stromal MMP-2 expression was associated significantly with high stage $(P=0.01)$, high 
grade $(P=0.03)$, presence of ascites $(P<0.01)$, suboptimal cytoreduction $(P=0.01)$, and positive lymph node status $(P<0.01$; Table 2$)$.

Some level of MMP-9 expression was detected in the cytoplasm of majority of the samples. Ninety-seven percent ( 87 of 90) of the samples showed high epithelial MMP-9 expression (moderate or strong) and 3\% (3 of 90) were negative. Within this group, patients with moderate or lower (OS $\leq 2)$ expression of epithelial MMP-9 were compared to those with strong $(\mathrm{OS}=3$ ) expression. Stromal MMP-9 expression was detected in 86 of 90 specimens, with $70 \%$ of tumors exhibiting high stromal MMP-9 levels. In $60 \%$ of the tumors, the leading edge (invasive front) was associated with greater stromal MMP-9 expression. High epithelial MMP-9 expression did not correlate with any of the prognostic variables.

However, high expression of stromal MMP-9 was associated significantly with high stage $(P$ $<0.01)$, high grade $(P=0.02)$, presence of ascites $(P<0.01)$, serous histology $(P<0.01)$, suboptimal cytoreduction $(P=0.01)$, and positive lymph node status $(P<0.01$; Table 2$)$.

All specimens showed epithelial MT1-MMP expression (moderate in 56\% and strong expression was seen in 44\%). Stromal MT1-MMP was expressed in 87 of 90 specimens. Sixty-two percent of tumors showed low stromal MT1-MMP expression and 38\% showed high expression. The presence of both highly expressed epithelial or stromal MT1-MMP was associated with high stage $(P \leq 0.01)$, high grade $(P=0.01)$, presence of ascites $(P \leq$ $0.01)$, and positive lymph node status $(P<0.01)$. In addition, high expression of epithelial MT1-MMP was also associated with the development of distant metastasis $(P=0.01)$ and serous histology $(P=0.02$; Table 2$)$.

\section{Clinical outcome based on MMP expression and clinical variables}

Prior to testing the prognostic relevance of MMPs, we first did univariate analyses of traditional clinical variables for DSS. Of the 90 patients, there were 55 deaths $(61 \%)$ with a median overall survival of 4.08 years from time of diagnosis. Of these, 52 deaths $(94.5 \%)$ were attributable to EOC with a median DSS that was identical to overall survival (4.08 years). All further analyses done for DSS represent similar outcomes for OS in this cohort of patients. As expected, high stage $(P<0.01)$, high tumor grade $(P=0.03)$, serous histology $(P<0.01)$, presence of ascites $(P<0.01)$, suboptimal cytoreduction $(P<0.01)$, positive pelvic lymph node status $(P<0.01)$, and the presence of distant metastases $(P=0.02)$ were all significantly associated with a shorter DSS. Age was analyzed as a continuous variable and for each additional year of age, there was a 3\% increased risk of death from ovarian cancer $(P=0.01)$.

Based on the associations of MMP expression with aggressive tumor features, we next examined its effects on the clinical outcome of patients with EOC. Analyzing the data based on a Cox proportional hazards regression model (24), patients with tumors exhibiting high expression of epithelial and stromal MMP-2 $(P<0.01)$, MMP-9 $(P \leq 0.01)$, and MT1-MMP $(P<0.01)$ were each significantly associated with a shorter median DSS (Table 3$)$. Figure 2 depicts the Kaplan-Meier survival curves for MMP-2, MMP-9, and MT1-MMP.

Next, we did a tree-structured analysis for DSS to determine whether any specific combinations of MMP expression would confer the worst clinical outcome. Stratification of the study group based on MMP expression is shown in Fig. 3A. Patients with moderate (OS = 2) epithelial MT1-MMP and low (OS = 0 or 1) stromal MMP-9 expression (group I) had the best prognosis (median DSS was not reached; Fig. 3B). Patients with moderate epithelial expression of MT1-MMP and high (OS = 2 or 3) stromal MMP-9 expression (group II) had an intermediate DSS [median DSS, 5.75 years; 95\% confidence interval (CI), 2.9, not applicable]. These patients had a 14.3-fold risk of death from EOC as compared with the first group (HR, 14.3; 95\% CI, 3.22-36.2). Finally, patients with strong (OS = 3) epithelial 
expression of MT1-MMP (group III) had the shortest 5-year DSS (median DSS, 2.5 years; $95 \%$ CI, 1.54-3.80). The risk of death from EOC in this group was $\sim 36$-fold higher compared with group I (HR, 35.9; 95\% CI, 8.24-156.2). The difference in DSS between these groups was highly significant $(P<0.01)$.

In order to assess whether there was an independent association between any of the clinicopathologic variables and DSS, we did a multivariate Cox proportional hazards model. The initial model included multiple prognostic variables such as age, preoperative CA-125 level, stage, grade, ascites, cytoreduction, distant metastasis, as well as epithelial and stromal expression levels for MMP-2, MMP-9, and MT1-MMP. Nodal status was excluded from this model as 49 out of 90 (54.4\%) of the patients did not undergo lymph node evaluation. We used the backward stepwise elimination method for selecting significant predictors of DSS to generate the final Cox proportional hazards model (Table 4). Using this method, high stage $(P=0.04)$, high expression of stromal MMP-9 $(P=0.01)$, and high expression of epithelial $(P=0.01)$ and stromal $(P=0.04)$ MT1-MMP were associated with shorter DSS (Table 4).

To confirm our results regarding the significance of MMP variables, as determined by multivariate analysis, an additional likelihood ratio test was done. A hypothetical "best" model without the MMP variables was obtained by initially including all prognostic variables other than MMPs (i.e., age, CA125, stage, grade, ascites, presence of distant metastasis, and cytoreduction) and then performing stepwise backward selection. In this model, stage (HR, 4.62; 95\% CI, 1.34-15.94; $P=0.01$ ) and ascites (HR, 2.73; 95\% CI, 1.1$6.79 ; P=0.03$ ) were independent predictors of DSS. When MMP variables (epithelial and stromal expression levels of MMPs 2, 9 and MT1-MMP) are added to this best model, the MMPs listed in Table 4 remained statistically significant $(P<0.0001)$, further validating our results.

\section{Discussion}

The main findings of the present study are that high expression of MMP-2, MMP-9, and MT1-MMP, in particular by stromal cells, is significantly associated with poor prognosis in patients with EOC. Specifically, overexpression of MMP-9 and MT1-MMP by stromal cells was an independent predictor of short DSS among all the MMPs studied. These results indicate that contribution of peritumoral stromal cells to overall MMP expression is an important predictor of clinical outcome in epithelial ovarian cancer.

MMPs have emerged as key molecules involved in both local growth and distant metastasis in a variety of tumors. MMPs are enzymes that are able to degrade most components of the ECM (such as collagen, laminin, fibronectin, elastin and the protein core of proteoglycans; refs. 5, 25). They facilitate the interaction of tumor cells with its local microenvironment, which is a critical step in the subsequent development of metastasis (26). Although previous studies have evaluated the production of these proteases by tumor cells, our data along with other immunohistochemical analyses have revealed that MMPs are detected not only in tumor cells, but also in stromal cells $(21,27,28)$. Peritumoral stromal cells have been shown to be the predominant source of MMP-1, MMP-3, MMP-11, and MMP-2 in several tumors including breast, colon, head and neck, lung, and ovarian cancers $(9,28-31)$. We investigated the clinical relevance of three MMPs (MMP-2, MMP-9, and MT1-MMP) that have been consistently associated with ovarian cancer.

MMP-2 has been shown to be the primary gelatinolytic MMP that is secreted by ovarian cancer cells $(32,33)$. As seen in our study, previous immunohistochemical studies have also reported that MMP-2 expression is localized to both tumor cells and stromal cells such as 
fibroblasts and vascular endothelial cells $(34,35)$. In a study focused on the role of MMP-2, Torng and colleagues found that stromal MMP-2 expression was an independent predictor of DSS for patients with endometrioid ovarian cancer, but not with serous histology $(21,36)$. Our findings indicate that stromal MMP-2 expression is indeed predictive of poor outcome in univariate analysis, but this effect was lost in multivariate analysis when other MMPs were included in the model. It has been shown that stromal cells such as fibroblasts at the invasive edge of ovarian tumors show a striking increase in mRNA for MMP-2 and its inhibitor, tissue inhibitor of metalloproteinase-2 $(35,37)$. The dual cellular origin of MMP-2 in EOC points to a role in local invasion and progression. Thus, MMP-2 may act on tumor cells and the ECM in an autocrine/paracrine fashion, stimulating invasion regardless of the source of MMP-2.

The activation of MMP-2 has been well studied. It is known that MMP-2 is released as a pro-form and activated through a unique multi-step pathway involving MT1-MMP (38) and their colocalization has been well documented $(14,39)$. Stromal cells may contribute to the process of invasion and metastasis through MT1-MMP-mediated activation of MMP-2 or directly through the actions of MT1-MMP on the ECM. In a mouse model of ovarian cancer, high levels of MT1-MMP mRNA were detected in higher grade tumors and in tumors with invasive features (40). Additionally, higher levels of stromal MT1-MMP mRNA were associated with larger tumors (40). Our results suggest that patients with strong tumor epithelial MT1-MMP expression had the worst prognosis with the shortest DSS.

The functional significance of host-derived MMPs has been defined by recent studies using transgenic mice. Huang and colleagues implanted human ovarian cancer cells into the peritoneal cavity of nude mice that lacked the gene for MMP-9 (MMP-9 ${ }^{-/-}$) or were wildtype (MMP- $9^{+/+}$; ref. 18). Tumor incidence and growth were significantly lower in mice lacking MMP-9, and these tumors had decreased microvessel density as well as decreased macrophage infiltration (18). Thus, stromal MMP-9 production by tumor-infiltrating macrophages plays a critical role in the progressive growth of ovarian carcinoma. Coussens and colleagues also showed that the predominant source of MMP-9 in a transgenic model of multi-stage squamous cell carcinoma was the neutrophils, macrophages, and mast cells, rather than the neoplastic cells themselves (41). These findings suggest a distinct role for the production of metastasis-associated proteins by nonmalignant host cells in cancer development and progression. The prognostic value of stromal MMP-9 expression in ovarian cancer was shown by Ozalp and colleagues, who showed that patients whose tumors had weak staining of stromal MMP-9 had a significantly longer survival compared with those with moderate or intense stromal MMP-9 expression (42). Our findings extend prior work in that stromal MMP-9 expression was an independent prognostic variable for patients with ovarian cancer.

The importance of the microenvironment at the tumor-host interface during metastasis is becoming increasingly appreciated. It has been shown that cocultivation of breast (43) or ovarian (44) cancer cells with stromal cells such as fibroblasts causes an increase in expression of MMP-1, MMP-2, and tissue inhibitor of metalloproteinase-2 in the culture supernatant, indicating that the interaction between tumor cells and fibroblasts may enhance fibroblast production of these MMPs. Although physical cell-cell contact may play an important role in up-regulating MMP expression by both tumor and stromal cells (45), the mechanism of tumor cell-mediated MMP production by stromal cells is not fully known. One of the proposed tumor cell-associated regulatory molecules is EMMPRIN (extracellular matrix metalloproteinase inducer), which is a tumor cell surface glycoprotein that stimulates MMP-2 and MMP-9, and its own expression in tumor stroma (46). In addition, these investigators found that increased EMMPRIN expression resulted in an immediate stimulation of vascular endothelial growth factor transcription and accompanying vascular 
endothelial growth factor protein production in tumor cells (47). Recent findings also suggest that MT1-MMP may directly stimulate vascular endothelial growth factor expression via the Src tyrosine kinase signaling pathway (48). Another group has reported on the possible role of thrombospondin-1 as an inducer of MMP-9 production by fibroblasts when cocultured with breast cancer cells (49). In addition, there are data to suggest that cytokines such as interleukin 1 and tumor necrosis factor- $\alpha$ produced by the tumor regulate MMP expression by peritumoral stromal cells (50). In this context, microarray studies may help in further elucidating the interactions between the tumor and stromal cells. Although the tumor-host interactions that result in MMP stimulation are still under investigation, it is apparent that the expression and regulation of MMP expression are complex events that reflect the dynamic nature of the tumor microenvironment. In view of these findings, in vivo studies using small interfering RNA directed to human- and murine-specific MMPs both in wild-type and MMP knockout animals could help further define the relative contribution of tumor-cell and stromal MMPs to ovarian cancer development and progression (51).

In summary, we have shown that MMP-2, MMP-9, and MT1-MMP are expressed by both epithelial and stromal cells. High expression of stromal MMPs is correlated with aggressive features in EOC. Notably, multivariate survival analysis, taking into account all of the prognostic variables studied, identified high stromal expression of MMP-9 and MT1-MMP to be independent predictors of DSS in patients with EOC. These results point to the functional contribution of host-derived MMPs in the pathogenesis of ovarian carcinoma as well as to the clinical outcome of patients with this deadly disease. Although the role of MMPs as therapeutic targets remains to be further defined, it is possible that broad MMP inhibitors (for example, Col-3; ref. 52) targeting both epithelial and stromal MMPs may be useful in controlling ovarian cancer vascularization and metastasis.

\section{Acknowledgments}

Grant support: Portions of this work were supported by the Bettyann Asche-Murray Fellowship Award (A.A. Kamat) and National Cancer Institute grants CA11079301 and CA10929801 and the University of Texas M.D. Anderson Cancer Center Specialized Programs of Research Excellence in Ovarian Cancer 1P50CA83639 (A.K. Sood).

\section{References}

1. Jemal A, Murray T, Ward E, et al. Cancer statistics, 2005. CA Cancer J Clin. 2005; 55:10-30. [PubMed: 15661684]

2. Ahmed FY, Wiltshaw E, A'Hern RP, et al. Natural history and prognosis of untreated stage I epithelial ovarian carcinoma. J Clin Oncol. 1996; 14:2968-75. [PubMed: 8918494]

3. Liotta LA, Rao CN, Barsky SH. Tumor invasion and the extracellular matrix. Lab Invest. 1983; 49:636-49. [PubMed: 6317982]

4. Werb Z. ECM and cell surface proteolysis: regulating cellular oncology. Cell. 1997; 91:439-42. [PubMed: 9390552]

5. Yurchenco PD, Schittny JC. Molecular architecture of basement membranes. FASEB J. 1990; 4:1577-90. [PubMed: 2180767]

6. Woessner, JF, Jr.. The matrix metalloproteinase family. In: Parks, WC.; Mecham, RP., editors. Matrix metalloproteinases. Academic Press; San Diego (CA): 1998. p. 1-14.

7. Liotta LA, Steeg PS, Stetler-Stevenson WG. Cancer metastasis and angiogenesis: an imbalance of positive and negative regulation. Cell. 1991; 664:327-36. [PubMed: 1703045]

8. Sakata K, Shigemasa K, Nagai N, Ohama K. Expression of matrix metalloproteinases (MMP-2, MMP-9, MT1-MMP) and their inhibitors (TIMP-1, TIMP-2) in common epithelial tumors of the ovary. Int J Oncol. 2000; 17:673-81. [PubMed: 10995877] 
9. Fishman DA, Bafetti LM, Banionis S, Kearns AS, Chilukuri K, Stack MS. Production of extracellular matrix-degrading proteinases by primary cultures of human epithelial ovarian carcinoma cells. Cancer. 1997; 80:1457-63. [PubMed: 9338470]

10. Stack MS, Ellerbroeck SM, Fishman DA. The role of proteolytic enzymes in the pathology of epithelial ovarian carcinoma [review]. Int J Oncol. 1998; 12:569-76. [PubMed: 9472094]

11. Wu X, Li H, Kang L, Wang W, Shan B. Activated matrix metalloproteinase-2-a potential marker of prognosis for epithelial ovarian cancer. Gynecol Oncol. 2002; 84:126-34. [PubMed: 11748988]

12. Davidson B, Goldberg I, Gotlieb WH, et al. High levels of MMP-2, MMP-9, Mt1-MMP and TIMP-2 mRNA correlate with poor survivals in ovarian carcinoma. Clin Exp Metastasis. 1999; 17:799-808. [PubMed: 11089877]

13. Soto H, Seiki M. Membrane-type matrix metalloproteinases (MT-MMPs) in tumor metastasis. J Biochem. 1996; 119:209-15. [PubMed: 8882706]

14. Strongin AY, Collier IE, Bannikov G, et al. Mechanism of cell surface activation of 72-kDa type IV collagenase. Isolation of the activated form of the membrane metalloprotease. J Biol Chem. 1995; 270:5331-8. [PubMed: 7890645]

15. Davidson B, Goldberg I, Berner A, et al. Expression of membrane-type 1, 2 and 3 matrix metalloproteinases messenger RNA in ovarian carcinoma cells in serous effusions. Am J Clin Pathol. 2001; 115:517-24. [PubMed: 11293899]

16. Davidson B, Reich R, Berner A, et al. Ovarian carcinoma cells in serous effusions show altered MMP-2 and TIMP-2 mRNA levels. Eur J Cancer. 2001; 37:2040-9. [PubMed: 11597382]

17. Egeblad M, Werb Z. New functions for the matrix metalloproteinases in cancer progression. Nat Rev Cancer. 2002; 2:161-74. [PubMed: 11990853]

18. Huang S, Arsdall MV, Tedjarati S, et al. Contributions of stromal metalloproteinase-9 to angiogenesis and growth in human ovarian carcinoma in mice. J Natl Cancer Inst. 2002; 94:113442. [PubMed: 12165638]

19. Soini Y, Hurskainen T, Hoyhtya M, Oikarinen A, Autio-Harmainen H. 72 KD and 92 KD type IV collagenase, type IV collagen, and laminin mRNAs in breast cancer: a study by in situ hybridization. J Histochem Cytochem. 1994; 42:945-51. [PubMed: 8014478]

20. Nomura H, Fujimoto N, Seiki M, Mai M, Okada Y. Enhanced production of matrix metalloproteinase 2 (gelatinase A) in human gastric carcinomas. Int J Cancer. 1996; 69:9-16. [PubMed: 8600068]

21. Torng P, Mao L, Chan W, Huang S, Lin C. Prognostic significance of stromal metalloproteinase-2 in ovarian adenocarcinoma and its relation to carcinoma progression. Gynecol Oncol. 2004; 94:559-67. [PubMed: 14766248]

22. Sood AK, Fletcher MS, Gruman LM, et al. The paradoxical expression of maspin in ovarian carcinoma. Clin Cancer Res. 2002; 8:2924-32. [PubMed: 12231537]

23. LeBlanc M, Crowley J. Relative risk trees for censored survival data. Biometrics. 1992; 48:41125. [PubMed: 1637970]

24. Cox DR. Regression models and life tables (with discussion). JR Stat Soc B. 1972; 34:187-220.

25. Russel, P. Surface epithelial-stromal tumors of the ovary. In: Kurman, RJ., editor. Blaustein's pathology of the female genital tract. 4th ed.. Springer; NewYork: 1994. p. 705-82.

26. Curran S, Murray GJ. Matrix metalloproteinases in tumor invasion and metastasis. J Pathol. 1999; 189:300-8. [PubMed: 10547590]

27. Nielsen BS, Timshel S, Kjeldsen S, et al. $92 \mathrm{kDa}$ type IV collagenase (MMP-9) is expressed in neutrophils and macrophages but not in malignant epithelial cells in human colon cancer. Int $\mathbf{J}$ Cancer. 1996; 65:57-62. [PubMed: 8543396]

28. Sood AK, Fletcher MS, Coffin JE, et al. Functional role of matrix metalloproteinases in ovarian tumor cell plasticity. Am J Obstet Gynecol. 2004; 190:899-909. [PubMed: 15118611]

29. Basset P, Wolf C, Chambon P. Expression of the stromelysin-3 gene in fibroblastic cells of invasive carcinomas of the breast and other human tissues: a review. Breast Cancer Res Treat. 1993; 24:185-93. [PubMed: 8435474]

30. Okada A, Bellocq JP, Rouyer N, et al. Membrane-type matrix metalloproteinase (MT-MMP) gene is expressed in stromal cells of human colon, breast, and head and neck carcinomas. Proc Natl Acad Sci USA. 1995; 92:2730-4. [PubMed: 7708715] 
31. Huang LW, Garrett AP, Bell DA, Welch WR, Berkowitz RS, Mok SC. Differential expression of matrix metalloproteinase- 9 and tissue inhibitor of metalloproteinase-1 protein and mRNA in epithelial ovarian tumors. Gynecol Oncol. 2000; 77:369-76. [PubMed: 10831344]

32. Davidson B, Goldberg I, Gotlieb WH, et al. The prognostic value of metalloproteinases and angiogenic factors in ovarian carcinoma. Mol Cell Endocrinol. 2002; 187:39-45. [PubMed: 11988310]

33. Fishman DA, Bafetti LM, Stack MS. Membrane-type matrix metalloproteinase expression and matrix metalloproteinase-2 activation in primary human ovarian epithelial carcinoma cells. Invasion Metastasis. 1996; 16:150-9. [PubMed: 9186550]

34. Hoyhtya M, Fridman R, Komarek D, et al. Immunohistochemical localization of matrix metalloproteinase 2 and its specific inhibitorTIMP-2 in neoplastic tissues with monoclonal antibodies. Int J Cancer. 1994; 56:500-5. [PubMed: 8112886]

35. Westerlund A, Apaja-Sarkkinen M, Hoyhtya M, et al. Gelatinase A-immunoreactive protein in ovarian lesions-prognostic value in epithelial ovarian cancer. Gynecol Oncol. 1999; 75:91-8. [PubMed: 10502432]

36. Autio-Harmainen H, Karttunen T, Hurskainen T, et al. Expression of 72 kilodalton type IV collagenase (Gelatinase A) in benign and malignant ovarian tumors. Lab Invest. 1993; 69:312-21. [PubMed: 8377473]

37. Naylor MS, Stamp GW, Davies BD, Balkwill FR. Expression and activity of MMPs and their regulators in ovarian cancer. Int J Cancer. 1994; 58:50-6. [PubMed: 8014015]

38. Afzal S, Lalani EN, Poulsom R, et al. MT1-MMP and MMP-2 mRNA expression in human ovarian tumors: possible implication for the role of desmoplastic fibroblasts. Hum Pathol. 1998; 29:155-65. [PubMed: 9490275]

39. Nagase H, Woessner JF Jr. Matrix metalloproteinases. J Biol Chem. 1999; 274:21491-4. [PubMed: 10419448]

40. Drew AF, Blick TJ, Lafleur MA, et al. Correlation of tumor- and stromal-derived MT1-MMP expression with progression of human ovarian tumors in SCID mice. Gynecol Oncol. 2004; 95:437-48. [PubMed: 15581944]

41. Coussens LM, Tinkle CL, Hanahan D, Werb Z. MMP-9 supplied by bone marrow-derived cells contributes to skin carcinogenesis. Cell. 2000; 103:481-90. [PubMed: 11081634]

42. Ozalp S, Tanir HM, Yalcin OT, Kabukcuoglu S, Oner U, Uray M. Prognostic value of matrix metalloproteinase-9 (gelatinase-B) expression in epithelial ovarian tumors. Eur J Gynaecol Oncol. 2003; 24:417-20. [PubMed: 14584660]

43. Saad S, Bendall LJ, James A, Gottlieb DJ, Bradstock KF. Induction of matrix metalloproteinases MMP-1 and MMP-2 by co-culture of breast cancer cells and bone marrow fibroblasts. Breast Cancer Res Treat. 2000; 63:105-15. [PubMed: 11097087]

44. Boyd RS, Balkwill FR. MMP-2 release and activation in ovarian carcinoma: the role of fibroblasts. Br J Cancer. 1999; 80:315-21. [PubMed: 10408832]

45. Himelstein BP, Canete-Soler R, Bernhard EJ, Muschel RJ. Induction of fibroblast $92 \mathrm{kDa}$ gelatinase/type IV collagenase expression by direct contact with metastatic tumor cells. J Cell Sci. 1994; 107:477-86. [PubMed: 8207074]

46. Tang Y, Kesavan P, Nakada MT, Yan L. Tumor-stroma interaction: positive feedback regulation of extracellular matrix metalloproteinase inducer (EMMPRIN) expression and matrix metalloproteinase-dependent generation of soluble EMMPRIN. Mol Cancer Res. 2004; 2:73-80. [PubMed: 14985463]

47. Tang Y, Nakada MT, Kesavan P, et al. Extracellular matrix metalloproteinase inducer stimulates tumor angiogenesis by elevating vascular endothelial cell growth factor and matrix metalloproteinases. Cancer Res. 2005; 65:3193-9. [PubMed: 15833850]

48. Sounni NE, Roghi C, Chabottaux V, et al. Up-regulation of vascular endothelial growth factor-A by active membrane type- 1 matrix metalloproteinase through activation of Src-tyrosine kinases. J Biol Chem. 2004; 14:13564-74. [PubMed: 14729679]

49. Wang TN, Albo D, Tuszynski GP. Fibroblasts promote breast cancer cell invasion by upregulating tumor matrix-metalloproteinase-9 production. Surgery. 2002; 132:220-5. [PubMed: 12219015] 
50. Chang C, Werb Z. The many faces of metalloproteases: cell growth, invasion, angiogenesis and metastasis. Trends Cell Biol. 2001; 11:S37-43. [PubMed: 11684441]

51. Landen CN Jr. Chavez-Reyes A, Bucanca C, et al. Therapeutic EphA2 gene targeting in vivo using neutral liposomal small interfering RNA delivery. Cancer Res. 2005; 65:6910-8. [PubMed: 16061675]

52. Syed S, Takimoto C, Hidalgo M, et al. A phase I and pharmacokinetic study of Col-3 (Metastat), an oral tetracycline derivative with potent matrix metalloproteinase and antitumor properties. Clin Cancer Res. 2004; 10:6512-21. [PubMed: 15475438] 

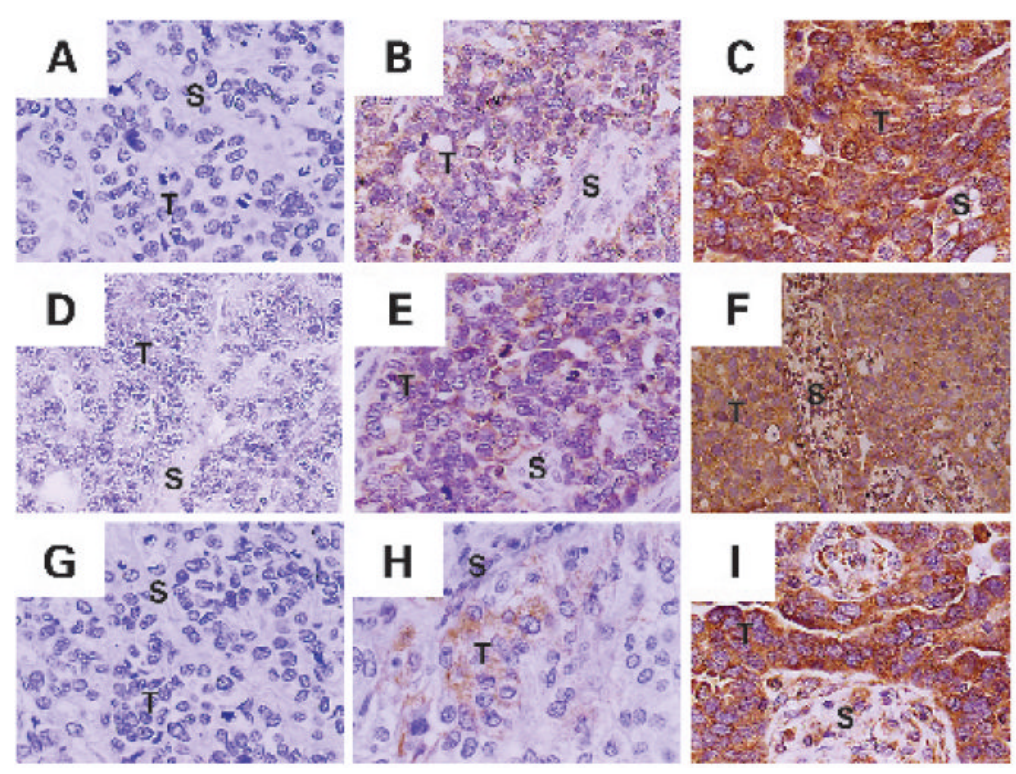

Fig. 1.

Immunohistochemical expression pattern of MMP-2, MMP-9, and MT1-MMP in epithelial ovarian carcinoma. Expression of MMP-2, MMP-9, and MT1-MMP was detected in both tumor epithelial cells $(T)$ and stromal cells $(S)$. $A-C$, negative, weak, and strong expression of MMP-2. $D-F$, negative, weak, and strong expression of MMP-9. $G-I$, negative, weak, and strong expression of MT1-MMP. Original magnification, $\times 200$. 

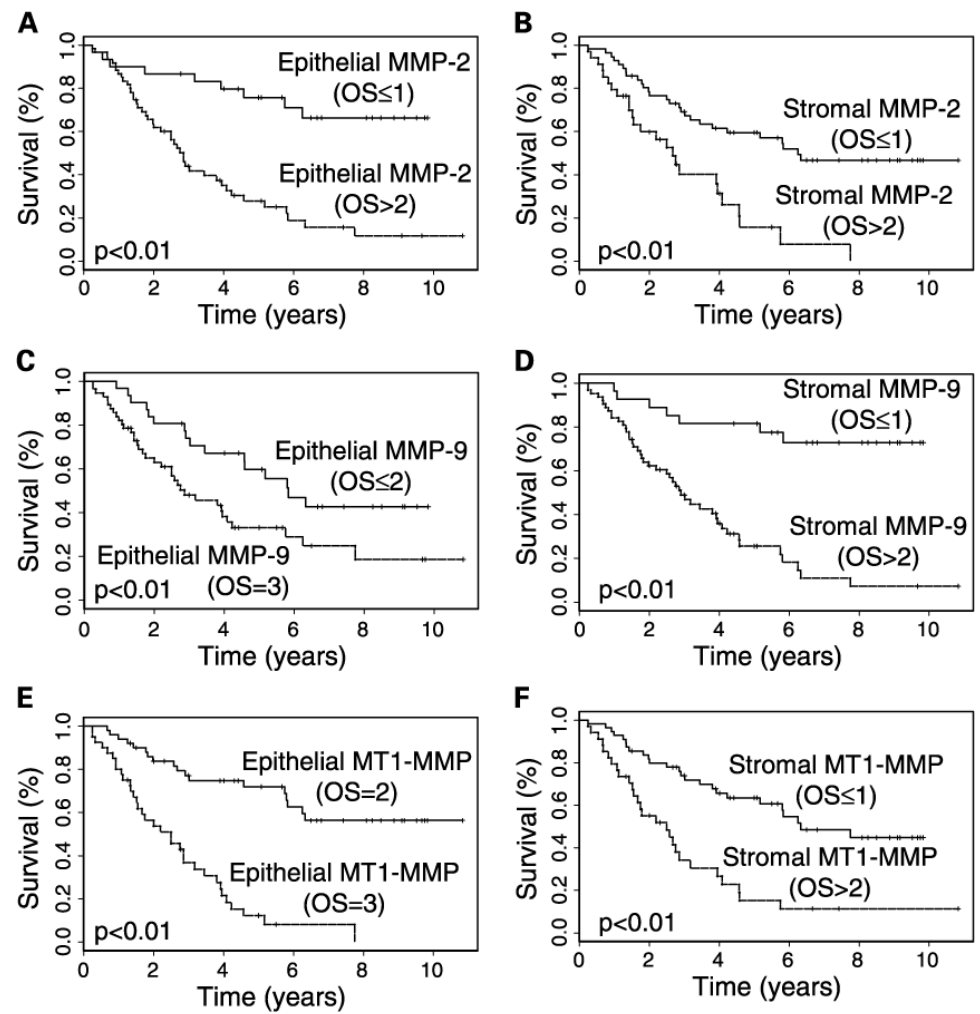

Fig. 2.

Kaplan-Meier survival curves for epithelial (left) and stromal (right) expression of MMP-2 $(A$ and $B)$, MMP-9 $(C$ and $D)$, and MT1-MMP $(E$ and $F$ ). In all categories other than epithelial MMP-2 and MMP-9, high (OS = 2 or 3) MMP expression was significantly associated with shorter DSS $(P<0.01)$. For epithelial MMP-2 and MMP-9, 97\% and 100\% of the samples, respectively, had high expression. For these two variables, patients with moderate or less $(\mathrm{OS} \leq 2)$ expression were compared with those with strong $(\mathrm{OS}=3)$ expression. 


\section{A}

Epithelial MT1-MMP $(O S=2)$

Epithelial MT1-MMP $(\mathrm{OS}=3)$

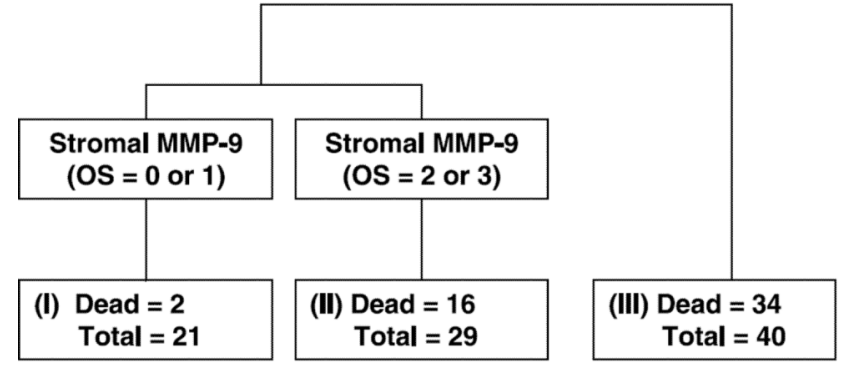

B

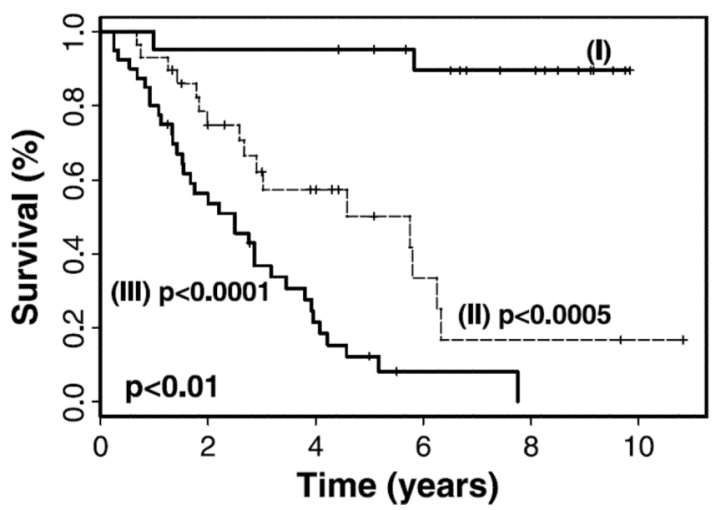

Fig. 3.

$A$, survival regression tree for all 90 patients with invasive epithelial ovarian carcinoma. The study group was divided into two distinct populations based on epithelial MT1-MMP expression. All samples had either moderate $(\mathrm{OS}=2)$ or strong $(\mathrm{OS}=3)$ epithelial MT1MMP expression. Within the group with moderate MT1-MMP expression, low (OS $=0$ or 1) or high ( $\mathrm{OS}=2$ or 3 ) stromal MMP-9 expression further stratifies the group. $B$, KaplanMeier survival curves for each node of the survival tree. Patients with strong expression of epithelial MT1-MMP had the shortest DSS, whereas those with moderate expression of epithelial MT1-MMP and low expression of stromal MMP-9 had the best outcome. The difference between the three groups was highly significant by log-rank test $(P<0.01)$. 
Table 1

Demographic features of patients with invasive epithelial ovarian cancer

\begin{tabular}{|c|c|}
\hline Variable & Number \\
\hline Mean age, y (range) & $59.3(34-83)$ \\
\hline Mean preoperative CA125 (IU/mL) & 1,716 \\
\hline Median (range) & $924(26-8,813)$ \\
\hline \multicolumn{2}{|l|}{ Stage } \\
\hline I & 11 \\
\hline II & 7 \\
\hline III & 56 \\
\hline IV & 16 \\
\hline \multicolumn{2}{|l|}{ Histologic subtype } \\
\hline Serous & 60 \\
\hline Other & 30 \\
\hline \multicolumn{2}{|l|}{ FIGO grade } \\
\hline Low (1 or 2$)$ & 37 \\
\hline High (3) & 53 \\
\hline \multicolumn{2}{|l|}{ Cytoreduction } \\
\hline Optimal & 63 \\
\hline Suboptimal & 27 \\
\hline \multicolumn{2}{|l|}{ Ascites } \\
\hline Absent & 24 \\
\hline Present & 66 \\
\hline \multicolumn{2}{|l|}{ Node status } \\
\hline Positive & 14 \\
\hline Negative & 27 \\
\hline Not done & 49 \\
\hline \multicolumn{2}{|l|}{ Distant metastasis } \\
\hline Absent & 79 \\
\hline Present & 11 \\
\hline \multicolumn{2}{|l|}{ Status } \\
\hline Alive without disease & 25 \\
\hline Alive with disease & 10 \\
\hline Dead of disease & 52 \\
\hline Dead of other causes & 3 \\
\hline
\end{tabular}

Abbreviation: FIGO, International Federation of Gynecology and Obstetrics. 


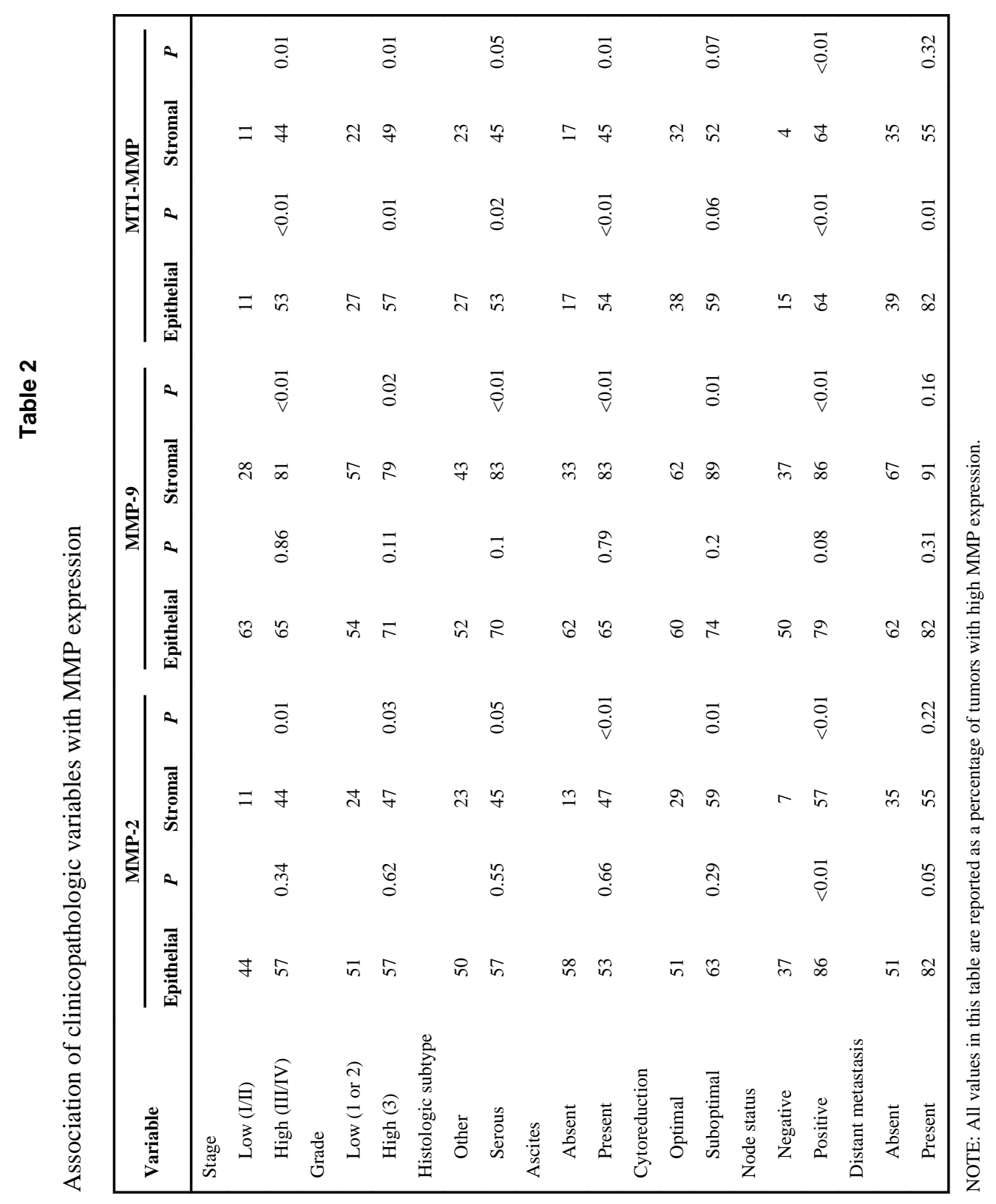

Clin Cancer Res. Author manuscript; available in PMC 2011 October 26. 


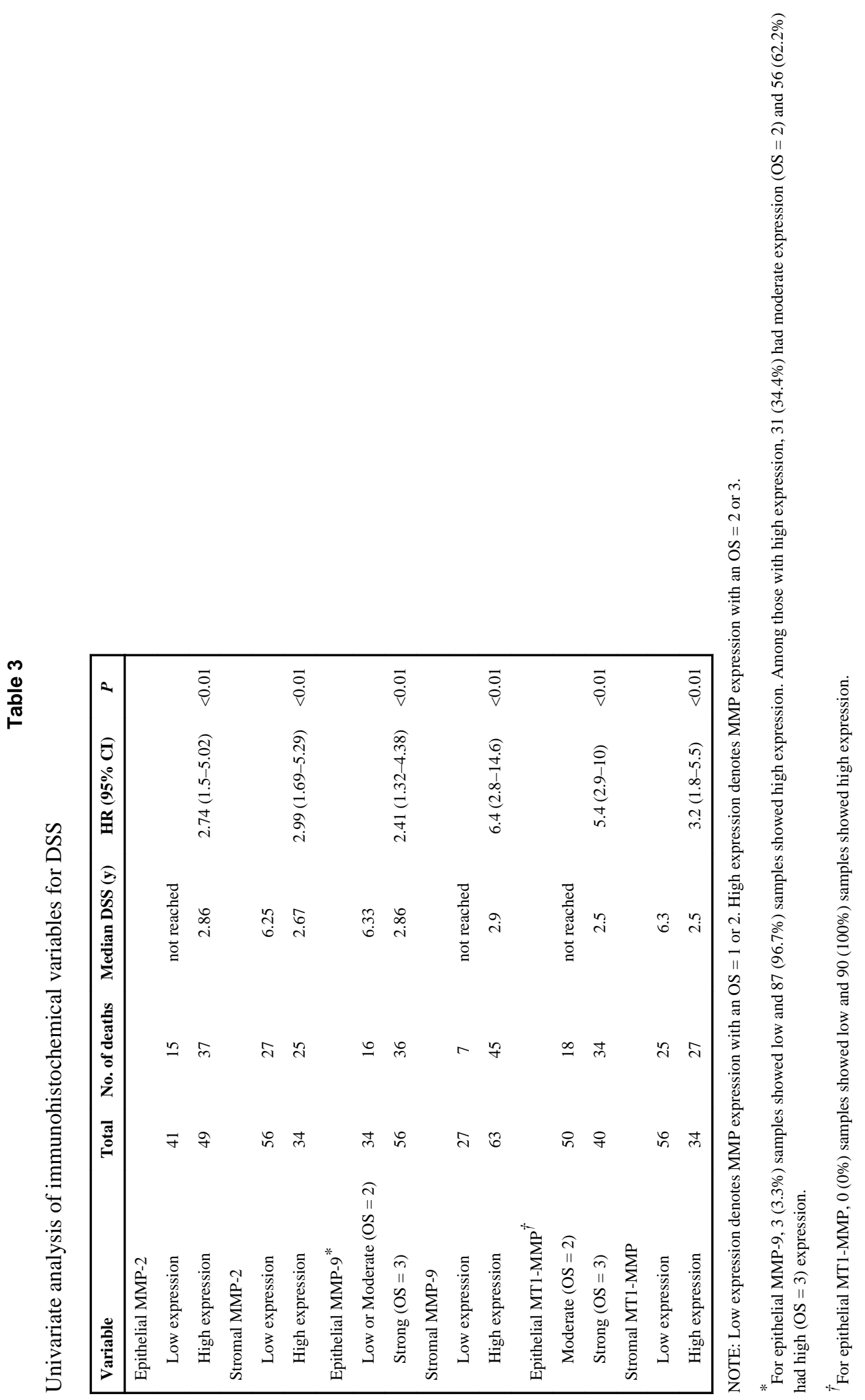

Clin Cancer Res. Author manuscript; available in PMC 2011 October 26. 
Table 4

Final multivariate Cox proportional hazards regression model for DSS using stepwise backward selection

\begin{tabular}{|lll|}
\hline Variable & HR $(\mathbf{9 5 \%}$ CI) & $\boldsymbol{P}$ \\
\hline High stage (III or IV) & $3.58(1.03-12.48)$ & 0.04 \\
High stromal MMP-9 expression & $3.33(1.37-8.11)$ & 0.01 \\
Strong epithelial MT1-MMP $($ OS $=3)$ & $2.52(1.30-4.88)$ & 0.01 \\
High stromal MT1-MMP expression & $1.87(1.03-3.39)$ & 0.04 \\
\hline
\end{tabular}

